\title{
ANTIPROTON ANNIHILATION INSIDE NUCLEI
}

\author{
M. CAHAY, J. CUGNON, P. JASSELETTE and J. VANDERMEULEN \\ Institut de Physique B5, University of Liege, \\ Sart Tilman, B-4000 Liege 1, Belgium
}

Received 5 April 1982

Revised manuscript received 25 May 1982

\begin{abstract}
Antiproton annihilation at the center of ${ }^{40} \mathrm{Ca}$ and ${ }^{108} \mathrm{Ag}$ nuclei is studied in the frame of an intranuclear cascade mode Primordial pions, issued from the annihilation, produce a density wave, which ejects about one fifth of the nucleons. The spectra of the emerging nucleons and of the produced pions are studied. For the latter, the spectrum deviates from a therm law.
\end{abstract}

It has recently been suggested [1] that the annihilation of slow antiprotons inside heavy nuclei provides a unique opportunity to study nuclear matter in unusual conditions. The deposit of an amount of $2 \mathrm{GeV}$ close to the center of the nucleus is expected to create a situation complementary to the one encountered in relativistic central nucleus-nucleus collisions. In the latter case, high temperature and high density are believed to appear after a fast compression stage whereas in the former case the transformation of two nucleon masses into kinetic energy may give rise to a high temperature, but would leave the density close to normal. Also, it has been suggested [1] that $\bar{p}$ annihilation at the center of the nucleus may provide the best example of a nuclear explosion where a strong disturbance would propagate radially from the center.

There exist old data $[2,3]$ on antiproton annihilation by nuclei, but they are too scarce to provide any useful indication on the plausibility of the above arguments. The advent in the next future of intense and monochromatic $\overline{\mathrm{p}}$ beams in the $0.1-2 \mathrm{GeV} / c$ momentum range at the LEAR facility at CERN will change the situation significantly. It has already prompted theoretical works, mainly in the direction of the evaluation of elastic and annihilation $\bar{p}-$ nucleus cross sections $[4,5]$.

Here, we study $\bar{p}$ annihilation at the center of ${ }^{40} \mathrm{Ca}$ and ${ }^{108} \mathrm{Ag}$ nuclei, by means of a cascade model. Although most of the annihilations are expected to take place at the surface, central annihilations are not unf quent. On the basis of experimental cross sections [ 6 . we estimate that $1 \%$ of the antiprctons in a $100 \mathrm{MeV}$ beam will reach the central region (3\% of the total vo ume) in the Ca nucleus; the fraction of central annihi lations increases with the beam energy. Note, howeve that potential effects could possibly change this estimate significantly [5].

Central events presumably correspond to a large fragment multiplicity (see ref. [1]) and below) and will presumably be distinguishable from the background. Our aim here is to provide a description of the main features of the evolution of the nucleus afte the $\bar{p}$ annihilation and to relate them to the argument mentioned above.

The method is similar to the one used previously $t$ study relativistic nucleus-nucleus collisions and described in detail in refs. $[7,8]$. It is sufficient here to say that the collision process is treated as a antinucleon-nucleon annihilation followed by a succession of on-shell relativistic classical hadron-hadron collisions involving pions, nucleons and $\Delta$-resonances with a non-zero width mass distribution and a finite lifetime. For every event, the $\bar{p}$ is assumed to annihilate with a nucleon at the center of the nucleus; the $\bar{p}$ has a residual laboratory kinetic energy of $100 \mathrm{MeV}$ ( $p_{\text {lat }}$ $=444 \mathrm{MeV} / c)$ and the Fermi motion of the nucleon $\mathrm{i}$ taken into account. A pion multiplicity $n$ is randomly chosen according to a gaussian law with $\langle n\rangle=5.08, \sigma$, 
$=1.07$, in agreement with a fit to low momentum $\mathrm{NN}$ annihilations by a statistical model [9]. The pion momenta are generated according to constant phase space density $[10,11]$, a reasonable approximation to the real situation [12]; for that purpose, the program FOWL [13] has been adapted and included in our code. Pure pionic annihilation is assumed; we neglect kaons in the annihilation, as well as in the subsequent cascade. It can be estimated on the basis of experimental branching ratios that about one kaon is produced for every 50 pions.

The interaction of the produced pions with a nucleon triggers a cascade, where the hadrons are allowed to interact in pairs and the $\Delta$ 's to decay. The following reactions are taken into account: $N+N \rightarrow N+N, N$ $+N \leftrightharpoons N+\Delta, N+\Delta \rightarrow N+\Delta, \Delta \rightleftharpoons \pi+N$; they are treated in the conditions described in refs. [7,8]; soft NN collisions $(\sqrt{s}<1925 \mathrm{MeV})$ are neglected. Also included here is a new feature in the $\pi-N$ interaction beyond the $\Delta$-resonance region: they are allowed to scatter elastically, with an angular distribution of the form $e^{a t}\left[a=5(\mathrm{GeV} / \mathrm{c})^{-2}\right]$, or inelastically, producing a $\pi \pi \mathrm{N}$ system according to constant phase space density. The cross sections are taken from ref. [14] and averaged over the isospin states.

Our first result deals with the absorption of pions. In the case of $40 \mathrm{Ca}$, an average number of 4.4 (for all charge states) leave the mucleus, which corresponds to an overall absorption of $13 \%$ (this figure rises to $20 \%$ in ${ }^{108} \mathrm{Ag}$ ). The evolution of the pion population is detailed in fig. 1, which shows the cumulated number of

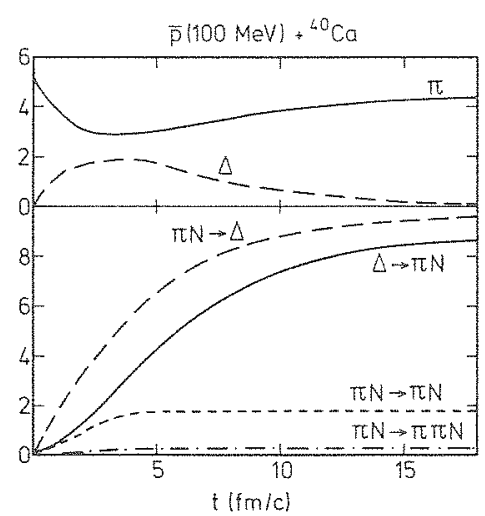

Fig. 1. Time evolution of the pion and $\Delta$ populations (upper part) and cumulated number of some reactions (lower part) for central annihilation of $\bar{p}$ in ${ }^{40} \mathrm{Ca}$ nuclei. The results correspond to an ensemble average over 900 runs. the different reactions controlling the pion abundance. In this energy range, the pions mainly interact through $\Delta$-formation and subsequent decays. After a time of $\sim 3 \mathrm{fm} / \mathrm{c}$, (the time $t=0$ coincides with the annihilation), all the initial pions are already transfomed into $\Delta$ 's. The absorption proceeds through the $\Delta N \rightarrow N N$ process. Let us mention that the absorption probability is consistent with early measurements [2], which however pertain to inclusive observations while our calculation deals with cases of deep nucleus penetration.

Our calculation is based on two-particle interac. tions; for the problem of pion reabsorption the procedure neglects the possibly important process of pion absorption on two nucleons [15].

The pions loose energy in their outward motion. Out of the initial $1925 \mathrm{MeV}$ carried by the primordial pions, $615 \mathrm{MeV}(854 \mathrm{MeV})$ are transferred to the nucleons of the ${ }^{40} \mathrm{Ca}\left({ }^{108} \mathrm{Ag}\right)$ nucleus. The primordial pions appear pretty much thermalized in the pp system, which moves with a velocity of $\beta=0.43$ in the laboratory system; the temperature is of the order of $T=120 \mathrm{MeV}$, in agreement with the equipartition of the available energy. The energy spectrum of the final pions looks quite isotropic in a frame moving with a velocity $\beta=0.1$. In that frame, the spectrum is close to a thermal one, except for the high energy tail (see fig. 2). The latter is presumably a remnant of the high en. ergy part of the primordial pions, which is not very

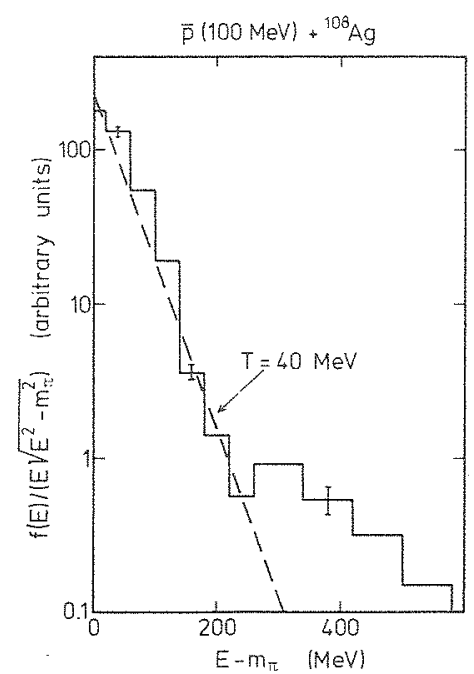

Fig. 2. Energy spectrum of the emitted pions in the $\bar{p}+{ }^{108} \mathrm{Ag}$ case. The error bars give the typical uncertainty of the calculation. 


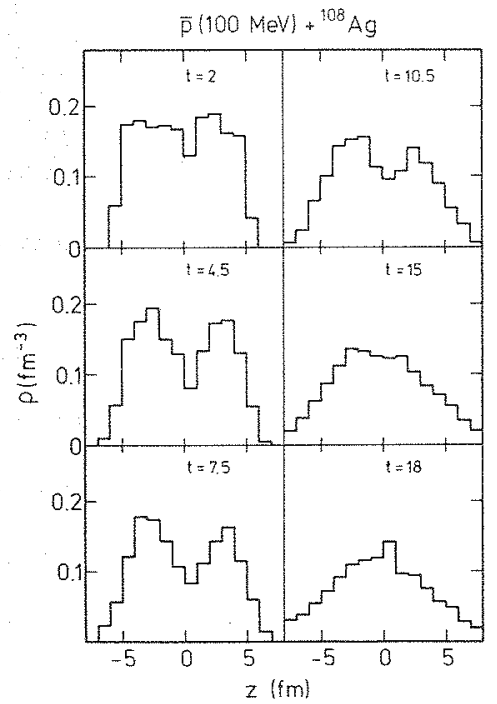

Fig. 3. Profiles of the nuclear density for different times (in $\mathrm{fm} / \mathrm{c}$ ) after the $\overline{\mathrm{p}}$ annihilation. The histograms represent the calculated density along the incident direction. The antiproton is assumed to come from the left.

much sensitive to the large $\pi \mathrm{N}$ resonant cross section and resists to full thermalisation. The low energy pions have a temperature of $T=53 \mathrm{MeV}$ for ${ }^{40} \mathrm{Ca}$ and $T \approx 40 \mathrm{MeV}$ for $108 \mathrm{Ag}$.

The pion flow gives rise to a perturbation in the density distribution of the nucleon system (see fig. 3); we call it a density wave for simplicity. A depression develops at the center of the nucleus, corresponding to the sweeping of the nucleons by the energetic outgoing pions. As a consequence, a slight compression is formed ahead of this disturbance. Due to the pressure generated, the central depression starts to fill up at $t$ $\approx 7.5 \mathrm{fm} / c$. At $t=18 \mathrm{fm} / c$, the density displays a maximum at the center of the nucleus. Meanwhile, the outgoing wave - certainly not a shock wave, which would imply a strong discontinuity - has ejected a certain fraction of the nucleons. For a ${ }^{108} \mathrm{Ag}$ target, around 21 nucleons are in this way removed from the initial Fermi sphere. Thus it is very likely that central annihilation will be characterized by high multiplicity events (with an isotropic distribution), as already suggested in ref. [1].

We finally say a few words about the distribution of the energy transferred to the participant nucleons (those having undergone at least one collision). The interesting result is that the angular distribution of these

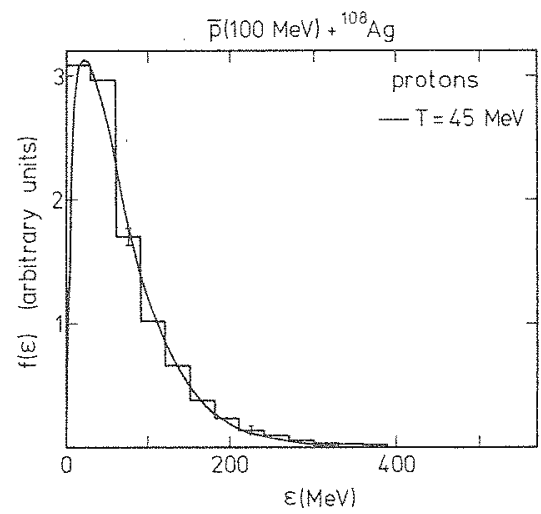

Fig. 4. Kinetic energy spectrum of the participant protons (see text). The full curve shows a fit by a Maxwell-Boltzmas distribution. The error bars give the typical uncertainty of th calculation.

nucleons is quite isotropic in the lab system and that the spectrum is very close to a thermal spectrum as $d$ picted by fig. 4 . The characteristic temperature is clo to $T \approx 45 \mathrm{MeV}$ for ${ }^{108} \mathrm{Ag}$ and $T \approx 60 \mathrm{MeV}$ for ${ }^{40} \mathrm{Ca}$, i.e. very close to the pion temperature. It seems that kind of equilibrium is reached in the exchange of ene gy between the pions and the participant nucleons. Once again, the $\Delta$-formation and the subsequent decay are largely responsible for this situation, since thi are the most important elementary processes (see fig. 1).

Due to the neglect of pion absorption on two nucleons, our results must be considered as a lower estimate of the amount of energy (and pressure) transferred from the annihilation zone to the surrounding nuclear matter.

In summary, the annihilation of an antiproton in the central zone of the nucleus seems to give rise to a rather complex interaction process: a density wave seems to develop through the nucleus; it ejects only a limited number of nucleons from the Fermi sphere without bringing strong density perturbation in the whole nucleus. In spite of the limitation in the number of involved nucleons, these appear surprisingly well thermalized.

Note that our results rely basically on the adopted picture of the annihilation. We have assumed that the energy liberated by this process is concentrated on a few pions of small size. As a consequence, a limited but not small number of nucleons will be disturbed. Also, the pion spectrum will present a tail which de- 
viates from a thermal law. If, on the contrary, the energy released by the initial annihilation is not concentrated on small volumes (those of the pions), more collective phenomena may develop. Therefore, a larger number of nucleons will receive energy from the primordial pions, as it is the case in an hydrody. namical approach, and a lower nucleon temperature is expected to be reached. Hence, it seems possible that the number of nucleons of high velocity and the spectrum of pions will reflect the manner on which the primordial energy is located.

Let us mention that the $\bar{p}$-nucleus interaction has been studied in an hydrodynamical model [16]. Although this calculation is not directly comparable with ours (in ref. [16]), only surface annihilation is studied), both agree on the fact that no shock wave is produced.

We are currently applying our method to the general case, where no restriction is placed on the $\bar{p}$ annihilation, in order to determine how the features of the process are modified.

\section{References}

[1] J. Rafelski, Phys. Lett. 91B (1980) 281.

[2] E. Segre, Ann. Rev. Nucl. Sci. 8 (1958) 127.

[3] A.G. Ekspong, A. Frisk, S. Nilsson and B.E. Ronne, Nucl. Phys. 22 (1961) 353.

[4] E.H. Auerbach, C.B. Dover and S.H. Kahanna, Phys. Rev. Lett. 46 (1981) 702.

[5] N.J. DiGiacomo, J. Phys. G7 (1981) L169.

[6] Particle Data Group (1972), $\overline{\mathrm{N} N}$ and $\overline{\mathrm{ND}}$ interactions - a compilation, LBL-58; CERN-HERA (1979), Compilation of cross sections, $p$ and $\overline{\mathrm{p}}$ induced reactions.

[7] J. Cugnon, T. Mizutani and J. Vandermeulen, Nucl. Phys. A352 (1981) 505.

[8] J. Cugnon, D. Kinet and J. Vandermeulen, Nucl. Phys., to be published.

[9] J. Vandermeulen, Lett. Nuovo Cimento 11 (1974) 243; 28 (1980) 60.

[10] E. Fermi, Prog. Theor. Phys. 5 (1950) 570.

[11] P.P. Srivastava and G. Sudarshan, Phys. Rev. 110 (1958) 765.

[12] C. Ghesquiere, in: Symp. on Antinucleon-nucleon interactions, CERN yellow report $74-18$, p. 436; L. Montanet, private communication.

[13] F. James, CERN yellow report 68-15

[14] CERN-HERA 79-01 (1979), compilation of cross sections I. $\pi^{-}$and $\pi^{+}$induced reactions.

[15] C.H.Q. Ingram, Nucl. Phys. A374 (1982) $319 \mathrm{c}$.

[16] D. Strottman, Los Alamos preprint (1981). 